\title{
A cytosolic oxygenase activity involved in the bioactivation of 2-aminofluorene
}

\author{
M. Leist*, A.D. Ayrton and C. Ioannides \\ Molecular Toxicology Research Group, Division of Toxicology, School of Biological Sciences, University \\ of Surrey, Guildford, Surrey, GU2 SXH (U.K.)
}

\section{Summary}

The contributions of the hepatic microsomal and cytosolic fractions in the metabolic activation of the promutagen 2-aminofluorene into mutagenic intermediates in the Ames test were investigated. Rat hepatic postmitochondrial, microsomal and cytosolic preparations could convert 2-aminofluorene to mutagens, the postmitochondrial preparation being the most and cytosol the least efficient. Pretreatment of the rats with Aroclor 1254 markedly enhanced the cytosol-mediated mutagenicity of the amine but increased microsomal- and postmitochrondrial-mediated mutagenicity only modestly. The cytosolmediated mutagenicity of 2-aminofluorene was abolished by heat treatment and by incubation with proteinase $\mathrm{K}$, but was unaffected by dialysis emphasising the protein nature of the cytosolic activation system. Oxygen radical generating systems and oxygen radical scavengers did not significantly influence the cytosol-mediated mutagenic response. Similarly incorporation of xanthine or allopurinol into the cytosolic activation system did not modulate the mutagenic response indicating no role for the molybdenum oxygenases. The cytosolic activation of 2 -aminofluorene differed from that mediated by the microsomes in cofactor requirement, substrate specificity and sensitivity to DMSO and (+)-catechin. Further centrifugation of the cytosolic fraction to remove any microsomal contamination did not decrease the cytosolic activation of 2-aminofluorene. It is concluded that the hepatic cytosol contains an oxygenase activity capable of activating certain aromatic amines.

Key words: 2-Aminofluorene; Aromatic amines; Bioactivation; Mutagenesis; Ames test

\section{Introduction}

The bioactivation of chemical carcinogens involves, in most cases, an initial oxidation to yield a reactive intermediate, the proximate carcinogen which can either interact with DNA and/or be further metabolised to an even more reactive species, the ultimate carcinogen, that very readily interacts with DNA, initiating the carcinogenic process. By far the most efficient tissue in the metabolic activation of

Correspondence to: C. Ioannides, Molecular Toxicology Research Group, Division of Toxicology, School of Biological Sciences, University of Surrey, Guildford, Surrey, GU2 5XH, U.K.

*Present Address: Universitat Konstanz, Fakultat fur Biologie, Lehrstuhlf, Biochem. Pharmakologie, Postfach 5560, D-7750, Konstanz, Germany. 
chemical carcinogens is the liver. Two enzyme systems appear to be responsible for the oxidation of carcinogens, both residing in the endoplasmic reticulum, namely: (a) The cytochrome $P$-450-dependent mixed-function oxidases, a superfamily of proteins with different substrate specificity [1] and (b) The FAD-monooxygenase system, primarily involved in the oxidation of aromatic amines [2]. The proximate carcinogens generated by these two enzyme systems may be further activated by various microsomal and cytosolic conjugases, e.g. acetylase, $\mathrm{N}, \mathrm{O}$-acyl transferase and sulphotransferase.

The cytosol is believed to be devoid of any role in the initial oxidation of chemical carcinogens and to be involved exclusively in the further metabolism of the oxidation products produced by microsomes. Incorporation of the cytosolic fraction into the microsomal activation system in the Ames test can markedly enhance the mutagenic effect of chemicals such as benzo[a]pyrene, aflatoxin $B_{1}$ [3], 2-aminofluorene [4], 2-acetylaminofluorene [5], 2-amino-3-methylimidazo-(4,5-f)quinoline [6], benzidine [7] and $N$-nitrosopiperidine [8].

In the early 1980's it was repeatedly reported that hepatic cytosolic preparations from Aroclor 1254-treated rats, in the absence of microsomes, could convert 2-aminofluorene, its acetylated derivative and 2-aminoanthracene to mutagens in the Ames test $[5,9,10]$. The same system, however, could not activate ethidium bromide, benzo $[a]$ pyrene and dimethylaminoazobenzene indicating that this cytosolic system may be specific to certain amines. Moreover, cytosolic enzymes could dealkylate dimethyl and phenylmethyl nitrosamines [11]. Despite the important implications of these observations in chemical carcinogenesis, this pathway has not been further investigated.

In the present paper we describe further studies establishing the enzymic nature of the cytosolic activating system and provide strong evidence that the activity is exclusively associated with the cytosol and is highly unlikely to be due to microsomal contamination.

\section{Materials and methods}

2-Aminofluorene and allopurinol (Aldrich Chemical Co., Gillingham, Dorset, U.K.), 4-aminobiphenyl (Phase Separations Ltd., Queensferry, Flints.), Aroclor 1254 (Robens Institute, University of Surrey,), proteinase K (Boehringer, Lewes, East Sussex), DMSO (FSA, Loughborough, Leics.), 2-acetylaminofluorene, 2,7-diaminofluorene, benzidine, $(+)$-catechin, xanthine, glucose 6-phosphate dehydrogenase and all cofactors (Sigma Co., Poole, Dorset) were purchased. The Salmonella typhimurium strain TA1538 was generously donated by Professor B.N. Ames, Berkeley, USA.

Male Wistar albino rats (150-200 g) were purchased from the Experimental Biology Unit, University of Surrey. Induction of the hepatic mixed-function oxidase system was achieved by a single intraperitoneal administration of Aroclor 1254 (500 $\mathrm{mg} / \mathrm{kg})$, dissolved in corn oil $(200 \mathrm{mg} / \mathrm{ml})$, the animals being killed on the 5 th day following administration. Hepatic postmitochondrial (S9), microsomal and cytosolic fractions were prepared as previously described [12].

The metabolic activation of the various promutagens was assessed using the Ames 
mutagenicity assay employing TA1538 as the indicator Salmonella typhimurium strain [13]. The activation system contained $10 \%(\mathrm{v} / \mathrm{v})$ of the appropriate hepatic fraction $(25 \% \mathrm{w} / \mathrm{v})$, supplemented with glucose 6-phosphate dehydrogenase ( 1 unit/plate) when isolated microsomes or heat/proteinase-treated cytosol were used. All promutagens were dissolved in DMSO and care was taken so that the total content of DMSO was constant and never exceeded $100 \mu \mathrm{l}$ per incubation. Where these conditions were altered, the changes are described in the appropriate legends.

\section{Results}

2-Aminofluorene was activated to mutagenic intermediates by all three control enzyme systems, namely $\mathbf{S} 9$, isolated microsomes and cytosolic fraction, $\mathbf{S} 9$ being the most and cytosol the least efficient (Fig. 1). Pretreatment with Aroclor 1254 markedly enhanced the cytosolic activation of 2-aminofluorene (about 4-fold at the highest mutagen concentration) and increased modestly activation by isolated microsomes, only at the highest carcinogen concentration, and to an even lesser extent activation by S9 (about 1.5-fold at the highest carcinogen concentration). With Aroclor 1254-induced preparations, the mutagenic response of 2-aminofluorene with cytosol employed as the activation system was about one third of that seen with microsomes only (Fig. 1). 2-Acetylaminofluorene was activated by all Aroclor 1254-induced hepatic fractions but not or very poorly by those from untreated animals, the $\mathrm{S} 9$ and microsomes being clearly the most effective (Fig. 2). With all activation systems 2-aminofluorene displayed mutagenicity that was much greater than that of its acetylated derivative.

Subsequent studies were conducted using 2-aminofluorene as the model mutagen since it was the most effectively activated by the cytosol among those studied. Initially the studies aimed at optimising the activation conditions. Incorporation of a preincubation step at $37^{\circ} \mathrm{C}$ enhanced the cytosol-induced mutagenicity of 2-aminofluorene, at least up to an hour (Fig. 3). When isolated microsomes were utilised as the activation system, maximum mutagenic response appears to have been reached following a $15 \mathrm{~min}$ preincubation period. Increasing the amount of cytosolic protein resulted in a similar rise in the 2-aminofluorene-mediated mutagenic response (Fig. 3). In marked contrast, increasing the microsomal protein content of the activation system decreased drastically the mutagenic response. In all subsequent studies $10 \%$ activation systems were employed since at this concentration good mutagenic responses were obtained with both hepatic fractions.

Using cytosol from Aroclor 1254-pretreated animals as activation system a positive mutagenic response was also seen with 2,7-diaminofluorene but not with either 4-aminobiphenyl or benzidine (Table I).

Both the microsomal and cytosolic fractions from Aroclor 1254-induced animals failed to activate 2-aminofluorene in the absence of any cofactors (Table II). However, when glucose 6-phosphate and NADP, or NADPH were incorporated into the hepatic fractions a strong mutagenic response was evident, particularly with the microsomes. Very weak mutagenic responses were seen, with both hepatic fractions, in the presence of NADP only, whereas in the presence of glucose 6-phosphate a weak response was seen with the microsomes but none with the cytosol (Table II). 

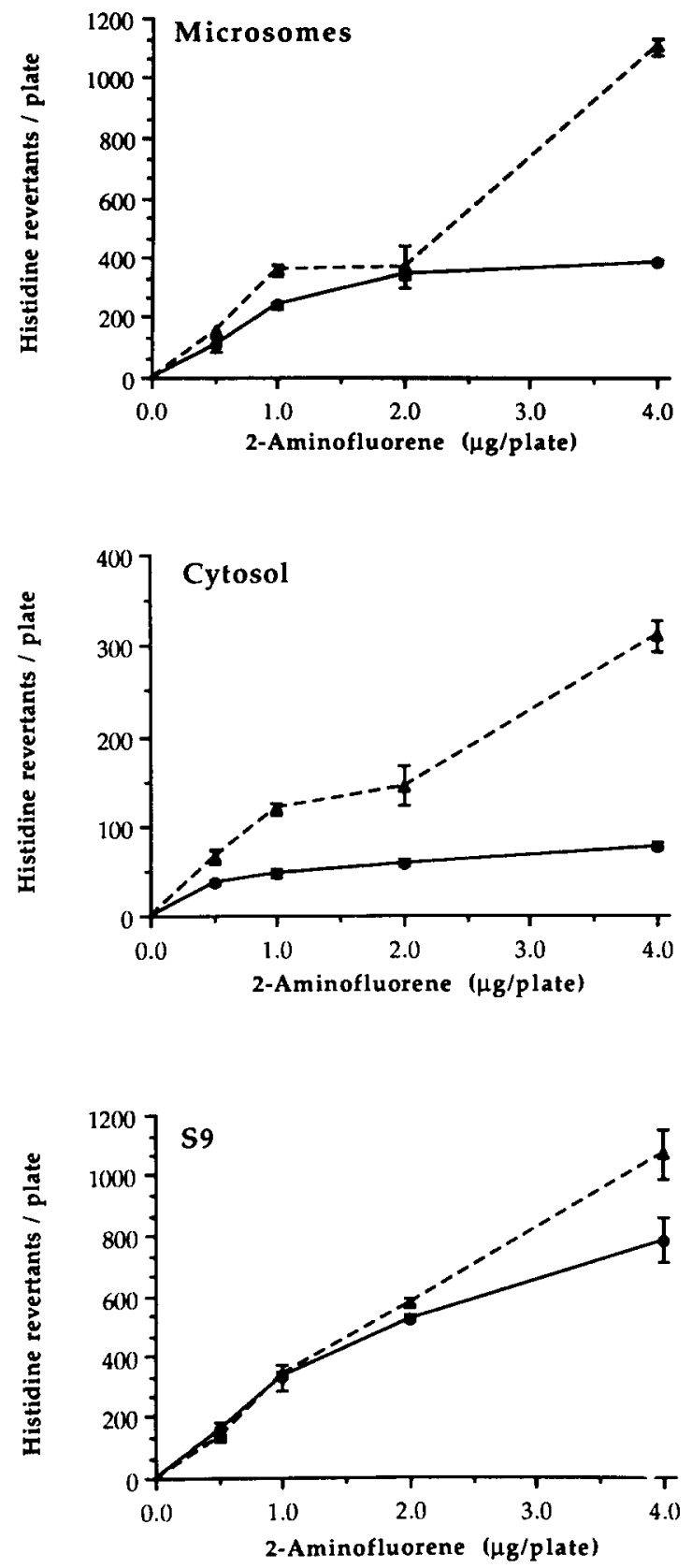

Fig. 1. Metabolic activation of 2-aminofluorene by hepatic preparations from control and Aroclor 1254-induced rats. The assay was carried out using the Salmonella typhimurium strain TA1538 and employing $10 \%(\mathrm{v} / \mathrm{v})$ activation systems. When microsomes were used, the activation system was supplemented with glucose 6-phosphate dehydrogenase ( 1 unit/plate). Each point represents the mean \pm S.D. for three experiments. The spontaneous reversion rate of $13 \pm 3$ has already been substracted. Control $(-)$, Aroclor 1254-treated (- - - -). 

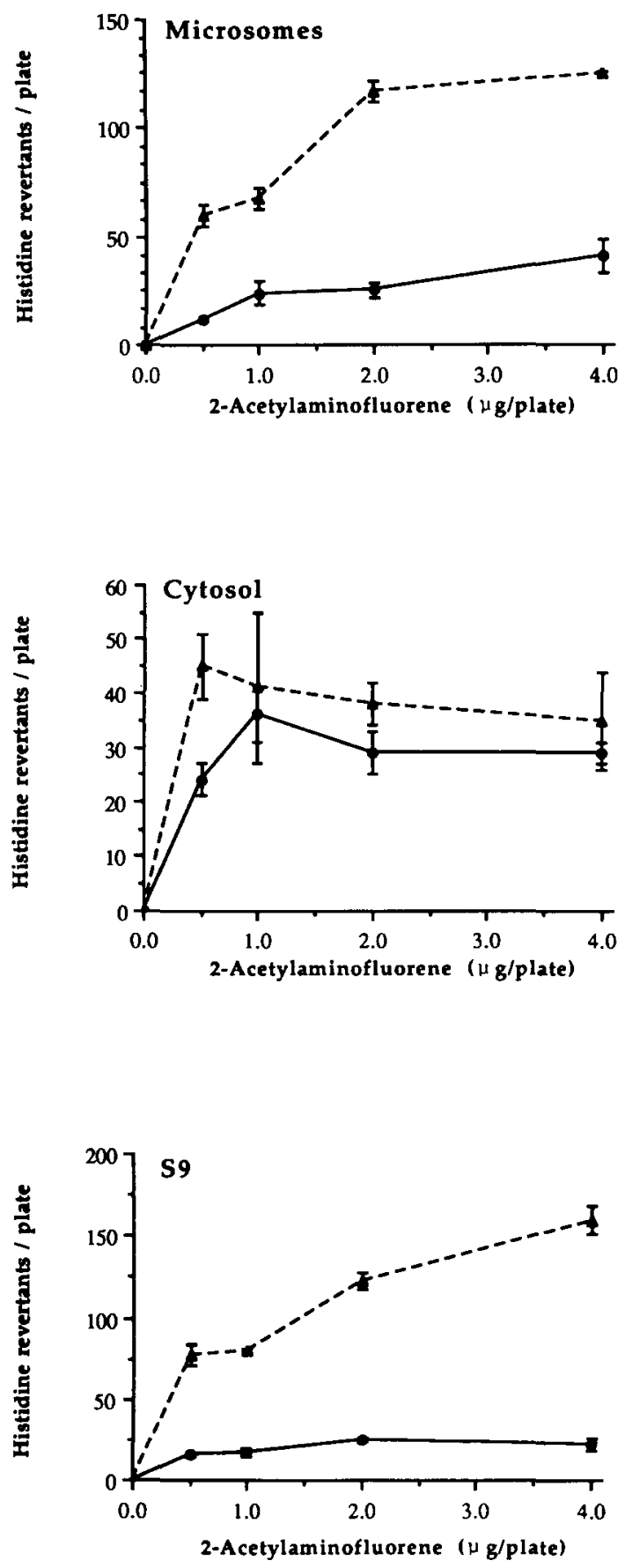

Fig. 2. Metabolic activation of 2-acetylaminofluorene by hepatic preparations from control and Aroclor 1254-induced rats. Conditions and symbols as in legend to Fig. 1. 

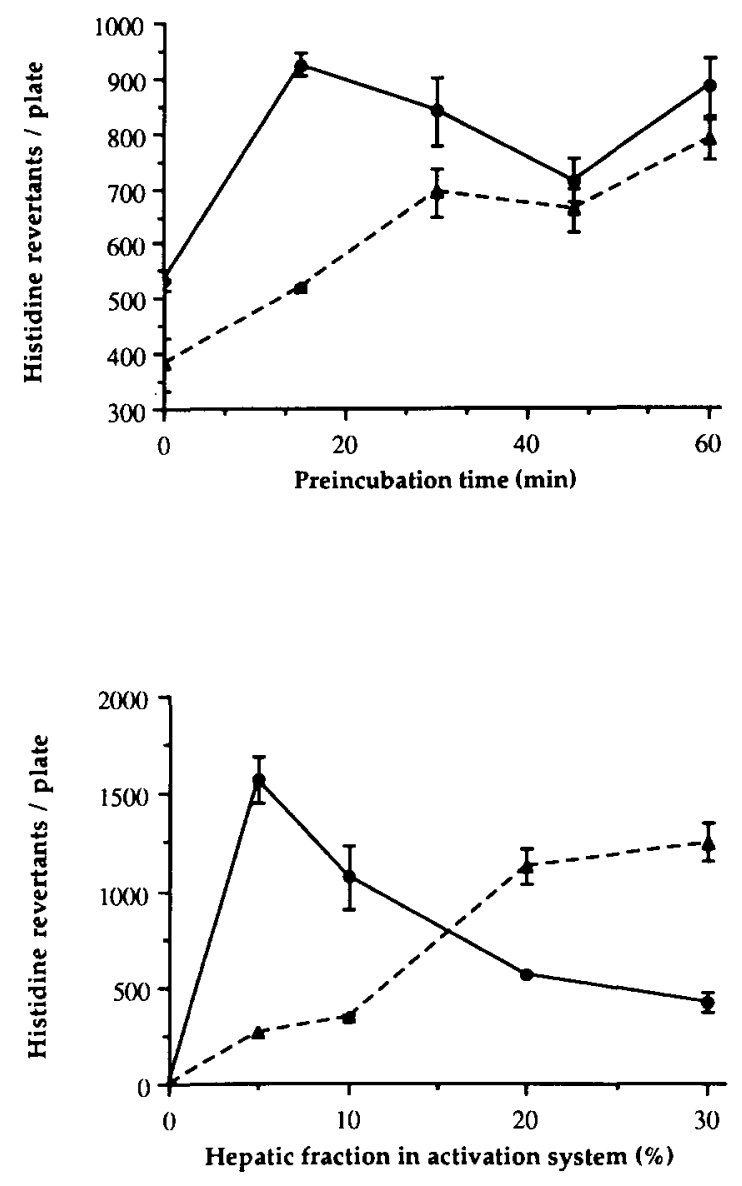

Fig. 3. Dependence of mutagenic response of 2-aminofluorene on the concentration of activation system and on the incorporation of a pre-incubation step. The test was performed using the Salmonella typhimurium strain TA1538, 2-aminofluorene ( $4 \mu \mathrm{g})$ and Aroclor 1254-induced rat hepatic preparations $(10 \%, v / v)$. When microsomes were used, the activation system was supplemented with glucose 6-phosphate dihydrogenase (1 unit/plate). Each point represents the mean \pm S.D. for three experiments. The spontaneous reversion rate of $12 \pm 1$ has already been substracted. Microsomes ( - ), cytosol $(--)$.

Interestingly NADH could efficiently support the microsomal, but not the cytosolic, activation of 2-aminofluorene. The role of NADH was further investigated to determine whether NADH and NADPH could elicit an additive or a synergistic response. Microsomal activation of 2-aminofluorene could be supported by NADH only to about $75 \%$ of that seen with NADPH, whereas only $10 \%$ of the cytosolic activation could be supported by NADH (Table II). Incorporation of both NADH and NADPH produced no additive or synergistic effect in either hepatic fraction. In order to evaluate the requirement for $\mathrm{O}_{2}$, the 30 min preincubation step was carried 
TABLE I

ACTIVATION OF VARIOUS AROMATIC AMINES BY AROCLOR 1254-INDUCED RAT HEPATIC CYTOSOL

Mutagenicity was determined using Salmonella typhimurium strain TA1538. The cytosolic fraction (25\%) was derived from the liver of rats pretreated with Aroclor 1254 and the final concentration in the activation system was $10 \%$. Activation system, bacteria and the amine (dissolved in $50 \mu$ I DMSO) were preincubated for $60 \mathrm{~min}$. The spontaneous reversion rate of $16 \pm 2$ has already been substracted. Results are presented as mean \pm S.D. of three experiments.

\begin{tabular}{lcc}
\hline Aromatic amine & $\begin{array}{l}\text { Concentration } \\
\text { (nmol/plate) }\end{array}$ & $\begin{array}{l}\text { Mutagenic response } \\
\text { (Histidine revertants/plate) }\end{array}$ \\
\hline 2-Aminofluorene & 11 & $628 \pm 25$ \\
& 22 & $760 \pm 13$ \\
4-Aminobiphenyl & 110 & $6 \pm 1$ \\
& 220 & $15 \pm 1$ \\
2,7-Diaminofluorene & 11 & $598 \pm 16$ \\
Benzidine & 22 & $816 \pm 20$ \\
& 110 & $2 \pm 1$ \\
& 220 & $2 \pm 1$ \\
\hline
\end{tabular}

out under a $\mathrm{N}_{2}$ atmosphere. In the absence of $\mathrm{O}_{2}$, the 2-aminofluorene-induced mutagenic response was reduced with both microsomal and cytosolic hepatic fractions, being more pronounced with the former. When 2-nitrofluorene was used, a mutagen that is not activated by oxidation but is reduced to the mutagenic hydroxylamine by bacterial nitroreductases, incubation in an $\mathrm{N}_{2}$ atmosphere did not influence the mutagenic response (Table II).

In order to eliminate any contribution of microsomal contamination to the cytosolic activation of 2-aminofluorene, the cytosol was further centrifuged twice and mutagenicity of the amine was investigated. No decrease in the mutagenicity was observed but, in contrast, a marked and reproducible increase in mutagenicity was evident (Table III). The cytosol-mediated mutagenicity of 2-aminofluorene was totally abolished by heat treatment and reduced to $10 \%$ following treatment with proteinase K (Table IV). A similar picture was obtained when microsomes served as the activation system. Storage of the cytosolic fraction at $4^{\circ} \mathrm{C}$ resulted in almost total loss of activity (Fig. 4). Dialysis of the cytosol against $10 \mathrm{mM}$ phosphate buffer (pH 7.5) plus 10\% glycerol did not alter 2-aminofluorene mutagenicity, being $325 \pm$ 45 before and $282 \pm 25$ after dialysis.

DMSO caused a marked, concentration-dependent decrease in the cytosolinduced mutagenicity of 2-aminofluorene (Fig. 5). When the promutagen was dissolved in a minimum of $20 \mu \mathrm{l}$ of the solvent, the mutagenic response was about four times higher than when 2-aminofluorene was dissolved in the standard volume of $100 \mu \mathrm{l}$. Under the same conditions, the microsomal activation of 2-aminofluorene was only slightly decreased by the increase in DMSO concentration, from $1365 \pm$ 
TABLE II

COFACTOR REQUIREMENT OF THE MICROSOMAL AND CYTOSOLIC ACTIVATION SYSTEMS OF 2-AMINOFLUORENE

Salmonella typhimurium TA1538, Aroclor 1254-induced hepatic fractions (10\%) and the carcinogen $(4 \mu \mathrm{g})$, dissolved in $100 \mu \mathrm{l} \mathrm{DMSO}$, were preincubated for $30 \mathrm{~min}$ at $37^{\circ} \mathrm{C}$ in a shaking waterbath. The spontaneous reversion rate of $6 \pm 1$ has already been substracted. Results are presented as mean \pm S.D. of at least three plates.

\begin{tabular}{llllr}
\hline Carcinogen & $\begin{array}{l}\text { Subcellular } \\
\text { fraction }\end{array}$ & $\begin{array}{l}\text { Cofactors and } \\
\text { final concentration } \\
\text { (mM) }\end{array}$ & $\begin{array}{l}\text { Gaseous } \\
\text { atmosphere }\end{array}$ & $\begin{array}{l}\text { Mutagenic response } \\
\text { (histidine } \\
\text { revertants/plate) }\end{array}$ \\
\hline 2-Aminofluorene & Microsomes & G-6-P* (5) + NADP (4) & $\mathrm{O}_{2}$ & $828 \pm 58$ \\
2-Aminofluorene & Microsomes & G-6-P (5) & $\mathrm{O}_{2}$ & $151 \pm 6$ \\
2-Aminofluorene & Microsomes & NADPH (8) & $\mathrm{O}_{2}$ & $855 \pm 13$ \\
2-Aminofluorene & Microsomes & NADP (4) & $\mathrm{O}_{2}$ & $39 \pm 10$ \\
2-Aminofluorene & Microsomes & NADH (8) & $\mathrm{O}_{2}$ & $442 \pm 18$ \\
2-Aminofluorene & Microsomes & None & $\mathrm{O}_{2}$ & 0 \\
2-Aminofluorene & Cytosol & None & $\mathrm{O}_{2}$ & 0 \\
2-Aminofluorene & Cytosol & G-6-P (5) + NADP (4) & $\mathrm{O}_{2}$ & $243 \pm 7$ \\
2-Aminofluorene & Cytosol & NADPH (8) & $\mathrm{O}_{2}$ & $255 \pm 14$ \\
2-Aminofluorene & Cytosol & G-6-P (5) & $\mathrm{O}_{2}$ & 0 \\
2-Aminofluorene & Cytosol & NADP (4) & $\mathrm{O}_{2}$ & $50 \pm 16$ \\
2-Aminofluorene & Cytosol & NADH (8) & $\mathrm{O}_{2}$ & $28 \pm 2$ \\
& & & $\mathrm{O}_{2}$ & $731 \pm 95$ \\
2-Aminofluorene & Microsomes & NADPH (8) & $\mathrm{O}_{2}$ & $549 \pm 42$ \\
2-Aminofluorene & Microsomes & NADH (8) & $804 \pm 28$ \\
2-Aminofluorene & Microsomes & NADH (8) + NADPH (8) & $\mathrm{O}_{2}$ & $222 \pm 10$ \\
2-Aminofluorene & Cytosol & NADPH (8) & $\mathrm{O}_{2}$ & $23 \pm 3$ \\
2-Aminofluorene & Cytosol & NADH (8) & $\mathrm{O}_{2}$ & $180 \pm 10$ \\
2-Aminofluorene & Cytosol & NADPH (8) + NADH (8) & $\mathrm{O}_{2}$ & $165 \pm \pm 19$ \\
& & & & $1657 \pm 23$ \\
2-Aminofluorene & Microsomes & NADP (4) + G-6-P (4) & $\mathrm{O}_{2}$ & $1224 \pm 60$ \\
2-Aminofluorene & Microsomes & NADP (4) + G-6-P (4) & $\mathrm{N}_{2}$ & $311 \pm 12$ \\
2-Aminofluorene & Cytosol & NADP (4) + G-6-P (4) & $\mathrm{O}_{2}$ & $568 \pm 11$ \\
2-Aminofluorene & Cytosol & NADP (4) + G-6-P (4) & $\mathrm{N}_{2}$ & $381 \pm 21$ \\
2-Nitrofluorene & Cytosol & NADP (4) + G-6-P (4) & $\mathrm{O}_{2}$ & $1650 \pm 19$ \\
2-Nitrofluorene & Cytosol & NADP (4) + G-6-P (4) & $\mathrm{N}_{2}$ & 1657 \\
\hline & & & & \\
\hline
\end{tabular}

${ }^{*} \mathrm{G}-6-\mathrm{P}=$ Glucose 6-phosphate

TABLE III

\section{EFFECT OF REPEATED CENTRIFUGATION ON THE CYTOSOLIC ACTIVATION OF 2-AMINOFLUORENE}

Aroclor 1254-induced cytosol (centrifugation 1) was furthe: centrifuged for two times at $105000 \times \mathrm{g}$ for $1 \mathrm{~h}$. The carcinogen $(4 \mu \mathrm{g})$, dissolved in DMSO $(100 \mu \mathrm{l})$, was preincubated with the bacteria and cytosol $(10 \%)$ for $60 \mathrm{~min}$ at $37^{\circ} \mathrm{C}$ in a shaking waterbath. The spontaneous reversion rate of $8 \pm 2$ has already been substracted. Results are presented as mean \pm S.D. for three experiments.

Number of centrifugations Histidine revertants/plate

\begin{tabular}{rr}
\hline 1 & 554 \\
464 & \pm 29 \\
2 & $1011 \pm 27$ \\
3 & \\
\hline
\end{tabular}


TABLE IV

EFFECT OF HEAT AND PROTEINASE DIGESTION ON THE MICROSOMAL AND CYTOSOLIC ACTIVATION OF 2-AMINOFLUORENE

2-Aminofluorene $(4 \mu \mathrm{g})$, bacteria and activation system $\left(10^{\%} \%\right)$ were preincubated for 60 min at $37^{\circ} \mathrm{C}$ in a shaking waterbath. Heat treatment consisted of heating the cytosol for $20 \mathrm{~min}$ at $60^{\circ} \mathrm{C}$ in a shaking waterbath. For proteinase digestion the cytosol was incubated with proteinase $\mathrm{K}(2 \mathrm{mg} / \mathrm{ml})$ for $60 \mathrm{~min}$ at $37^{\circ} \mathrm{C}$ in a shaking waterbath. Results are presented as mean \pm S.D. of 3 experiments. The spontaneous reversion rate of $15 \pm 3$ has already been substracted.

\begin{tabular}{llr}
\hline Subcellular fraction & Treatment & Histidine revertants/plate \\
\hline Microsomes & None & $1084 \pm 73$ \\
Cytosol & None & $325 \pm 45$ \\
Microsomes & Heat & $25 \pm 1$ \\
Cytosol & Heat & $6 \pm 1$ \\
& & \\
Microsomes & None & $655 \pm 18$ \\
Cytosol & None & $334 \pm 11$ \\
Microsomes & Proteinase K & $24 \pm 1$ \\
Cytosol & Proteinase K & $46 \pm 21$ \\
\hline
\end{tabular}

26 to $1180 \pm 35$ histidine revertants/plate, a reduction of only $14 \%$. Similarly, retinol decreased, in a concentration-dependent fashion, the cytosolic activation of 2-aminofluorene (Fig. 5). Finally, superoxide dismutase (50 units/plate), catalase (50 units/plate), mannitol $(50 \mathrm{mM})$, ascorbic acid $(10 \mathrm{mM}),(+)$-catechin $(1 \mathrm{mM})$ and the molybdenum oxygenase inhibitors xanthine $(300 \mu \mathrm{M})$ and allopurinol $(1 \mathrm{mM})$ had no significant influence on the cytosolic activation of the mutagen (results not shown).

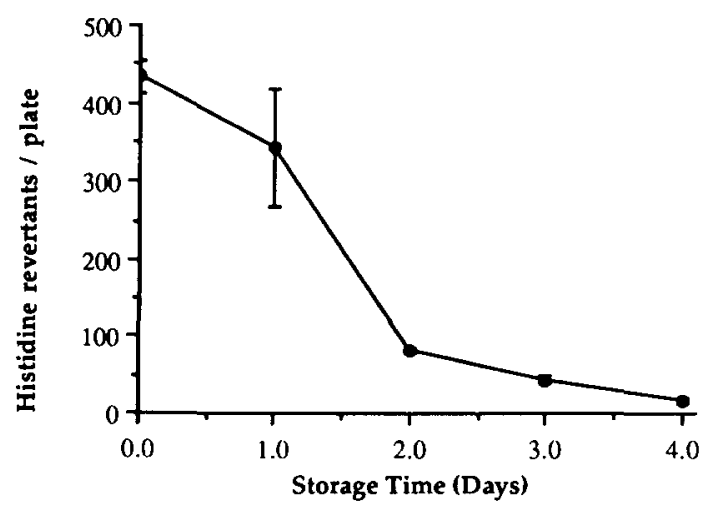

Fig. 4. Effect of storage on the cytosol-induced mutagenicity of 2-aminofluorene. The test was carried out using the Salmonella typhimurium strain TA1538, 2-aminofluorene $(4 \mu \mathrm{g})$ and Aroclor 1254-induced rat hepatic cytosol $(10 \%, \mathrm{v} / \mathrm{v})$. The cytosolic fraction was stored in a refrigerator for up to 4 days. Each point represents the mean \pm S.D. of three experiments. The spontaneous reversion rate of $12 \pm 3$ has already been substracted. 

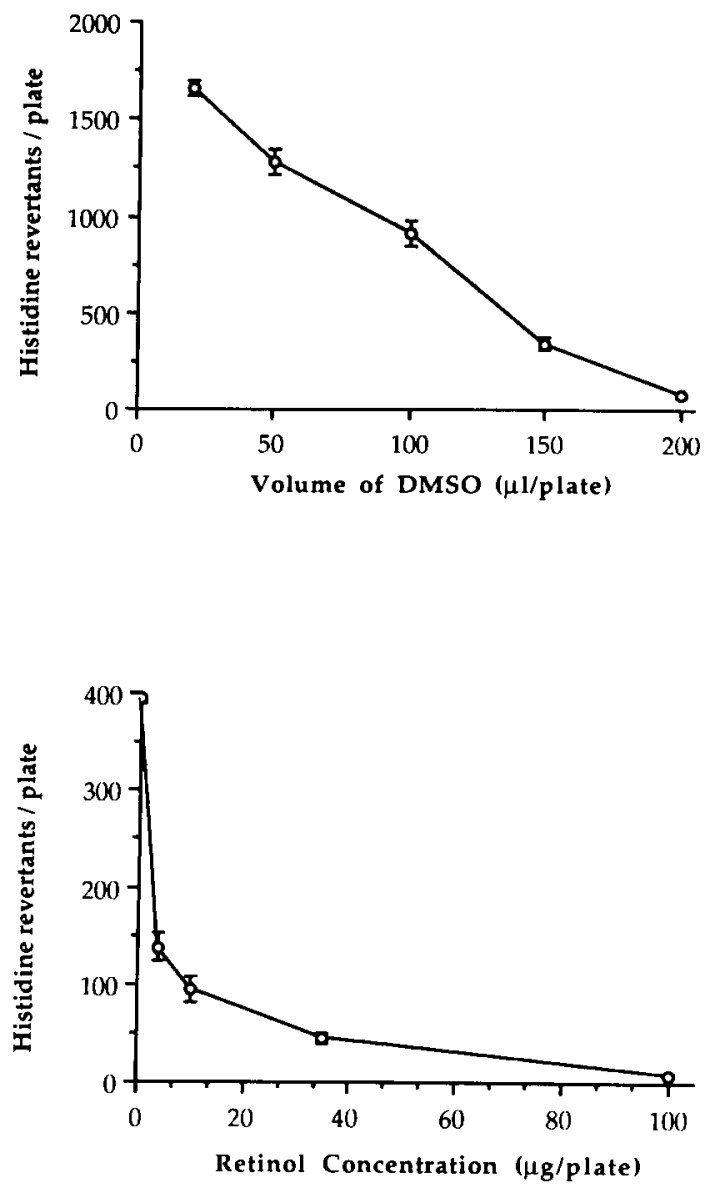

Fig. 5. Inhibition of the cytosol-induced mutagenicity of 2-aminofluorene by DMSO and retinol. The experiment was carried out using the Salmonella typhimurium strain TA1538, 2-aminofluorene $(4 \mu \mathrm{g})$ and hepatic cytosol $(10 \%, \mathrm{v} / \mathrm{v})$ from Aroclor 1254-induced rats. In the first study the total amount of DMSO in the activation system ranged from 20 to $200 \mu \mathrm{l}$. In the second study retinol (5-100 $\mu \mathrm{g} / \mathrm{plate})$ was added into the activation system. In both studies a pre-incubation step of $60 \mathrm{~min}$ at $37^{\circ} \mathrm{C}$ was incorporated. Each point represents the mean \pm S.D. of three experiments. The spontaneous reversion rates of $10 \pm 2$ and $7 \pm 1$ in the DMSO and retinol study respectively, have already been substracted.

\section{Discussion}

The first and rate limiting, step in the bioactivation of aromatic amines is believed to be an $\mathrm{N}$-hydroxylation catalysed by microsomal enzymes, the generated hydroxylamine serving as the proximate mutagen. Further metabolism by phase II conjugation enzymes eventually results in the production of ultimate electrophilic species such as the nitrenium ions that bind covalently to the DNA and initiate the carcinogenic process. The present paper describes a unique cytosolic oxygenase activity, 
apparently of high specific substrate specificity, which is effective in activating certain aromatic amines to mutagenic intermediates in the Ames test. As ringhydroxylated products are largely non-mutagenic $[14,15]$ it may be inferred that this enzyme catalyses the $\mathrm{N}$-hydroxylation of the amine. The requirements for oxygen and reduced dinucleotides also support such a role. Moreover, fluorene and 2-acetylfluorene, i.e. the parent compounds lacking the amino group, displayed no mutagenic response under these conditions (results not shown) indicating that arene oxides are unlikely to be involved in the mutagenic process.

The possibility that the cytosolic activity may simply reflect microsomal contamination has been raised [9]. In this paper we provide strong experimental evidence that this is not the case, and that the cytosolic activation is catalysed by a novel system. (a) Cofactor requirement differs in that NADH can clearly support the microsomal but not the cytosolic activation of 2-aminofluorene. (b) The Aroclor 1254-induced cytosol failed to convert 4-aminobiphenyl and benzidine to mutagenic intermediates, which are, however, activated by the microsomes $[7,14,16]$. It is worth pointing out that 2-aminoanthracene, frequently used as a positive control in mutagenicity studies, is also extensively metabolised by hepatic cytosolic fractions [17]. (c) Further centrifugation of the cytosolic fraction to remove any microsomal contamination present did not result in any decrease in the cytosolic activation of 2-aminofluorene but, in contrast, a reproducible increase in the mutagenic response, was seen. This increase may reflect the removal of material associated with microsomes that inhibits the cytosolic activity. (d) (+)-Catechin, an inhibitor of cytochrome $P-450$ reductase known to suppress the microsomal activation of aromatic amines, including 2-aminofluorene [18], failed to inhibit the cytosolmediated mutagenicity of 2-aminofluorene, (e) Cytosolic activation of 2-aminofluorene is very markedly suppressed by DMSO whereas the microsomal activation is only slightly inhibited. (f) When Aroclor 1254-induced hepatic preparations were utilised as activation systems for 2-aminofluorene, the cytosolic activation was $30 \%$ of that induced by the microsomes, incompatible with minor microsomal contamination of the cytosol. However, in this case, it may be argued that the mutagenic response induced by the cytosol reflects, at least partly, the further metabolism by phase II reactions of the primary metabolites to more mutagenic intermediates. As the activation systems were not supplemented with any phase II cofactors, the most likely action of the cytosol is to protect the oxidation of the $\mathrm{N}$ hydroxy-2-aminofluorene to the less mutagenic 2-nitrosofluorene [4]. A final, but unlikely possibility, which cannot be excluded is that an enzyme associated with the endoplasmic reticulum, but different to the one catalysing the microsomal activation of 2-aminofluorene, may be released into the cytosol during centrifugation.

Enzymes present in the cytosol that can play a role in the metabolism of xenobiotics are the molybdenum oxidases, aldehyde oxidase and xanthine oxidase. Aldehyde oxidase cannot be envisaged to contribute in the initial metabolism of 2-aminofluorene and moreover it appears not to be induced by Aroclor 1254. In the present study xanthine itself, as well as the potent inhibitor of xanthine oxidase allopurinol, failed to inhibit the cytosolic activation of 2-aminofluorene; furthermore, this enzyme has no requirement for NADPH. Clearly the molybdenum oxidases cannot account for the cytosol-induced mutagenicity of 2-aminofluorene. An 
enzyme system, present in the cytosol and capable of oxidising polycyclic aromatic hydrocarbons has also been described [19]. Since this system functions in the absence of added cofactors it may be inferred that it is different to the one described in the present study.

The cytosolic activation of 2-aminofluorene, similar to the microsomal activity, was heat labile and was almost totally abolished by treatment with proteinase $\mathrm{K}$. When stored at $4^{\circ} \mathrm{C}$, a complete loss of cytosolic activity was seen within 4 days. Finally, the cytosol-induced mutagenicity of 2-aminofluorene was unaffected by overnight dialysis against sodium phosphate buffer. Collectively, these observations testify to the protein nature of the cytosolic activation system. Cytosolic activation increased with increasing protein concentration, but not the microsomal activation, in agreement with previous studies [13].

Pretreatment of rats with Aroclor 1254, a mixture of polychlorinated biphenyls, an established inducer of the hepatic cytochrome $P-450$ families concerned with xenobiotic metabolism enhanced the cytosolic activation of 2-aminofluorene and its acetylated derivative indicating that the cytosolic activity is subject to induction by exposure to environmental chemicals. However, only a modest increase in mutagenic response was seen when S9 or microsomes were used as the activation system and only at the higher carcinogen concentrations. 2-Aminofluorene is selectively $\mathrm{N}$ hydroxylated and activated by the P450 I family and especially the A2 protein [20]. Moreover induction of this family by 2-aminofluorene appears to be an important factor determining its carcinogenicity [21], The lack of major effect in the microsome-induced mutagenicity of 2-aminofluorene following induction with Aroclor 1254 indicates that the P450 I family plays a limited role in the mutagenicity of this amine and suggests that other, constitutive proteins, non-inducible by Aroclor 1254, are largely responsible for the mutagenicity of this amine, or alternatively the FAD-monooxygenase system can account for all the activation. However, the possibility that Aroclor 1254 induces the deactivation of 2-aminofluorene through ring-hydroxylation to a much greater extent than $\mathrm{N}$-hydroxylation cannot be excluded.

The cytosolic activation of this amine required $\mathrm{O}_{2}$ and was inhibited when the preincubation was carried out in an atmosphere of nitrogen. The observation that considerable mutagenic response remained even when nitrogen was used is likely due to the fact that, when the agar containing the bacteria and activation system are plated, aerobic conditions are re-established, thus initiating the oxidative activation of the amine.

The marked, concentration-dependent inhibition of the cytosolic activation of 2-aminofluorene by DMSO and retinol, established oxygen radical scavengers, implicates oxygen radicals in the activation process. Oxygen radicals may be responsible for the oxidation and oxygenation of chemical carcinogens including 2-aminofluorene $[22,23]$. However, such a mechanism in the cytosolic activation of this amine is not supported by the experimental findings that superoxide dismutase, catalase and ascorbic acid did not influence mutagenicity. Moreover, substitution of the cytosol in the activation system with haem, haemoglobin, or the radicalgenerating xanthine/xanthine oxidase system resulted only in a marginal increase in the number of histidine revertants over spontaneous reversion rate (results not 
shown) and could not account for the observed cytosolic activation of 2-aminofluorene. These findings suggest that DMSO and retinol have a direct effect on the cytosolic enzyme, impairing its activity.

An interesting and unexpected observation was that the microsomal activation of 2-aminofluorene could be supported by NADH. Similar observations have been reported for other carcinogens such as nitrosopiperidine [8], dimethylnitrosamine [24] and the S9-mediated activation of 2-acetylaminofluorene [25]. It may be concluded that an NADH-dependent microsomal enzyme can catalyse the $\mathrm{N}$ oxygenation of 2-aminofluorene or, alternatively, certain cytochrome $P$-450 families may utilise NADH as the only electron donor. No synergistic or additive effect between NADH and NADPH was observed in the present study.

In summary, the present paper describes the presence of a cytosolic oxygenase activity that can convert certain aromatic amines to their genotoxic intermediates and may thus, play a role in the well known carcinogenicity of these chemicals.

\section{References}

1 C. Ioannides and D.V. Parke, The cytochrome P450 I gene family of microsomal hemoproteins and their role in the metabolic activation of chemicals. Drug Metab. Rev, 22 (1990) 1.

2 D.M. Ziegler, Flavin-containing mono-oxygenases: Catalytic mechanism and substrate specificities. Drug Metab. Rev., 19 (1988) 1.

3 G.T.P. Saccone and M.W. Pariza, Enhancement of hepatic microsome-mediated bacterial mutagenesis by the rat liver soluble protein fraction. Mutat. Res., 88 (1981) 135 .

4 G.T.P. Saccone, B.R. DasGupta and M.W. Pariza, Enhancement of $N$-hydroxy-2-aminofluorene bacterial mutagenicity by the soluble protein fraction from rat liver and partial purification of the enhancement activity. Cancer Res., 41 (1981) 4600.

5 R. Forster, M.H.L. Green and A. Priestly, Enhancement of S9 activation by S105 cytosolic fraction. Carcinogenesis, 2 (1981) 1081.

6 A. Abu-Shakra, C. Ioannides and R. Walker, Metabolic activation of 2-amino-3-methylimidazo(4,5- $f$ quinoline by hepatic preparations. - Contribution of the cytosolic fraction and its significance to strain differences. Mutagenesis, 1 (1986) 367.

7 J.N. Smith and C. Ioannides, Cytosolic potentiation of the rat hepatic microsome-mediated mutagenicity of benzidine. Mutagenesis, 2 (1987) 205.

8 A.D. Ayrton, J.N. Smith and C. Ioannides, Bioactivation of $N$-nitrosopiperidine to mutagens: role of hepatic cytochrome $P-450$ proteins and contribution of cytosolic fraction. Carcinogenesis, 8 (1987) 1691.

9 R. Forster, M.H.L. Green and A. Priestley, Apparent activation of 2-acetylaminofluorene and other aromatic amines by cytosolic preparations. Mutat. Res., 85 (1981) 187.

10 D.B. McGregor, M. McConville, C. Menzies and R.D. Prentice, Differing activation pathways for 2-acetylaminofluorene to a mutagen in vitro. Mutat. Res., 102 (1982) 39.

11 M.B. Kroeger-Koepke and C.J. Michejda, Evidence for several demethylase enzymes in the oxidation of dimethylnitrosamine and phenylmethylnitrosamine by rat liver fractions. Cancer Res., 39 (1979) 1587.

12 C. Ioannides and D.V. Parke, Mechanism of induction of hepatic drug metabolising enzymes by a series of barbiturates. J. Pharm. Pharmacol., 27 (1975) 739.

13 D.M. Maron and B.N. Ames, Revised methods for the Salmonella mutagenicity test. Mutat. Res., 113 (1983) 173.

14 H.A. Masson, C. Ioannides, J.W. Gorrod and G.G. Gibson, The role of highly purified cytochrome $P-450$ isozymes in the activation of 4-aminobiphenyl into mutagenic products in the Ames test. Carcinogenesis, 4 (1983) 1583.

15 S. Tong, J.N. Smith, D. Manson, J.W. Gorrod and C. Ioannides, The metabolic activation of 2-naphthylamine to mutagens in the Ames test. Anticancer Res., 6 (1986) 1107. 
16 C.M. Steele and C. Ioannides, Induction of rat hepatic mixed-function oxidases by aromatic amines and its relationship to their bioactivation to mutagens. Mutat. Res., 162 (1986) 41.

17 A.D. Ayrton, S. Neville and C. Ioannides, Cytosolic activation of 2-aminoanthracene: implications in its use as a diagnostic mutagen in the Ames test. Mutat. Res., In Press.

18 C.M. Steele, M. Lalies and C. Ioannides, Inhibition of the mutagenicity of aromatic amines by the plant flavonoid (+)-catechin. Cancer Res., 45 (1985) 353.

19 J.W. Flesher and S.R. Mayers, Oxidative metabolism of 7-methylbenz[a]anthracene 12-methylbenz[a]anthracene and 7,12-dimethylbenz[a]anthracene by rat liver cytosol. Cancer Lett., $26(1985) 83$.

20 G.J. Hammons, F.P. Guengerich, C.C. Weiss, F.A. Beland and F.F. Kadlubar, Metabolic oxidation of carcinogenic arylamines by rat, dog and human hepatic microsomes and by purified flavincontaining and cytochrome $P-450$ monooxygenases. Cancer Res., 45 (1985) 3578.

21 A.D. Ayrton, M. McFarlane, R. Walker, S. Neville and C. Ioannides, The induction of P450 I proteins by aromatic amines may be related to their carcinogenic potential. Carcinogenesis, 11 (1990) 803.

22 S.D. Aust, L.A. Morehouse and C.E. Thomas, Role of metals in oxygen radical reactions. J. Free Rad. Biol. Med., 1 (1985) 3.

23 G.F. Strniste, J.W. Nichols and R.T. Okinaka, Photochemical oxidation of 2-aminofluorene. Correlation between the induction of the direct-acting mutagenicity and the formation of nitro- and nitroso aromatics. Mutat. Res., 151 (1985) 15.

24 L.Y.Y. Fong, K.M. Lee and H.J. Lin, Mutagenesis in Salmonella after NADH-dependent microsomal activation of dimethylnitrosamine. Mutat. Res., 105 (1982) 29.

$25 \mathrm{~K}$. Takeishi, S. Okudo-Kaneda and T. Seno, Mutagenic activation of 2-acetylaminofluorene by guinea-pig liver homogenates: Essential involvement of cytochrome $P-450$ mixed-function oxidases. Mutat. Res., 62 (1979) 425. 